\title{
Primary lower segment caesarean section in multipara: materno-fetal outcomes
}

\author{
Grishma P. Agrawal*
}

Department of Obstetrics and Gynecology, Grant Government Medical College and JJ Group of Hospitals, Mumbai, Maharashtra, India

Received: 14 November 2018

Accepted: 06 December 2018

*Correspondence:

Dr. Grishma P. Agrawal,

E-mail: grishma.agrawal29@gmail.com

Copyright: () the author(s), publisher and licensee Medip Academy. This is an open-access article distributed under the terms of the Creative Commons Attribution Non-Commercial License, which permits unrestricted non-commercial use, distribution, and reproduction in any medium, provided the original work is properly cited.

\section{ABSTRACT}

Background: All-natural birth has a purpose and a plan; who would think of tearing open the chrysalis as the butterfly is emerging? Who would break the shell to pull the chick out?" Marie Mongon. It is of particular interest, in light of increased incidence of abdominal delivery throughout the country and in the world, to judge the validity of this procedure when used for the first time in the multipara.

Methods: All the cases of Primary caesarean delivery in multipara over a period of 18 months were studied with regards to the indication, associated risks factors, and perinatal morbidity and mortality. The primary objective of the study was to find out the status of primary Lower Segment Caesarean Section (LSCS) in multipara in tertiary care center.

Results: The overall incidence of LSCS was 30.7\%. The incidence of Primary LSCS in multipara was $23.4 \%$. The most common indication of LSCS was Foetal Distress i.e. 40.8\%. Almost all caesarean sections were done in Emergency (99.2\%). $12.3 \%$ of patients underwent PPH intra-operatively with $0.7 \%$ patient requiring Obstetric hysterectomy. Majority of the neonates were admitted in NICU for Respiratory Distress (30\%). Major cause of Postoperative morbidity was wound gape seen in $10.8 \%$ patients.

Conclusions: The fact that a multipara has had one or more vaginal deliveries should be regarded as an optimistic historical fact, not as diagnostic criteria for spontaneous delivery of the pregnancy at hand. Hence a multiparous woman in labour requires the same attention as that of primigravida and may still require a caesarean section for safe delivery. Good antenatal and intrapartum care and early referral will help reduce the maternal and perinatal morbidity.

Keywords: LSCS, Materno-fetal outcomes, Multipara

\section{INTRODUCTION}

The advent of better anaesthesia, availability of improvised surgical techniques and prophylactic antibiotics have made caesarean section a relatively safer and so common procedure. The decision whether to perform a caesarean section or not, is based on the individualized judgement of the obstetrician, the hospital where the caesarean would be performed. Economic factors and fear of litigation are other considerations which may indirectly influence such decisions. Multipara is woman who has completed/delivered 2 or more pregnancies after 20 weeks or more. Parity is determined by the number of pregnancies reaching 20 weeks and not by the number of the foetus delivered, so parity is the same for singleton pregnancy or multi-foetal delivery or delivery of live or stillborn infant. ${ }^{1}$ It is a common belief amongst public that once a mother delivers two children vaginally, all her subsequent deliveries will be vaginally. This results in poor obstetric care amongst multiparous mothers. ${ }^{2}$ In fact, multiparity is a problem associated with low age of marriage, illiteracy, low socio-economic 
status, preference for male child, high perinatal mortality and ignorance about family planning program. ${ }^{3,4}$ Primary caesarean section in multipara means first caesarean section done in the patients who had delivered vaginally two or more. In spite of vast literature dealing with problems of caesarean section in general, very little has been written concerning the problem of primary Caesarean section in multipara.

It is for these reasons that in present study the attention has been directed to the indication for caesarean section in women who have previously delivered vaginally. The present study focuses on the indication for caesarean section and the maternal and fetal outcome in multiparous women who had delivered vaginally earlier. The present analytical study attempts to critically analyze 130 cases of caesarean deliveries performed in tertiary hospital over a span of eighteen months with an aim to identify the indications and risk factors involved in early peri-natal morbidity and mortality.

\section{METHODS}

The total number of multipara cases i.e. previous two and more vaginal deliveries admitted to Antenatal ward and Labour ward of our Tertiary Care Institute, who underwent LSCS, over a period of 18 months were studied.

The obstetric data was analyzed, with all cases of Primary caesarean delivery in multipara being studied with regards to the indication, associated risks factors, their maternal outcome and perinatal morbidity and mortality. Detailed obstetric, medical history, clinical examination was recorded in Excel sheets. Indications and Intraoperative findings were noted. The decision to perform a caesarean section in each of these patients was made by a consultant on duty in consultation with the unit head telephonically. Post-operative maternal and neonatal follow up was done till patient was discharged from the hospital.

The primary objective of the study was to find out the status of primary LSCS in multipara in tertiary care center.

\section{Inclusion criteria}

- Multipara with previous two and more vaginal delivery in reproductive age group undergoing LSCS.

\section{Exclusion criteria}

- Previous LSCS

- Previous Hysterotomy

- Previous Myomectomy.

After applying the inclusion and exclusion criteria, total numbers of cases found during this period were 130 .
Caesarean section rate was calculated by using the formula

Caesarean section rate $=$ Total Number of Caesarean section x 100/Total Number of delivery.

Primary Caesarean section rate in multipara=Total Number of Primary Caesarean section in multipara x 100/ Total Number of Caesarean section in multipara.

We carried LSCS audit in the above period, analysed excel sheet and observed following parameters:

- Incidence

- Age

- Parity

- Registration status

- Booked status

- Gestational age

- Associated disorders

- Indications of LSCS

- Mode of identification of fetal distress

- Intra-operative findings

- Intra-operative complications

- Duration of surgery

- Birth weight in NICU

- Neonatal outcome

- Birth weight in NICU

- Early Perinatal Morbidity and Mortality.

- Relation of indications and high-risk factors with neonatal morbidity.

- Total hospital stay in days

- Post-operative complications.

\section{RESULTS}

Our observations are as follows:

\section{Incidence}

The study was carried out in Tertiary Care Institute over a period of 18 months. Combined numbers of deliveries from 1st February 2013 to 31st July 2014 were 8766. Out of which 2696 were LSCS. So, the overall incidence of LSCS is $30.7 \%$.

The total number of multipara delivered were 1869.Out of this population, multipara undergoing LSCS were 554.Out of this population, the multipara undergoing LSCS for the first time were 130 . So, the incidence of Primary LSCS in multipara is $23.4 \%$.

\section{Age}

In the present study, maximum cases were seen in the age group of 25 to 29 years of age i.e 54 cases (41.5\%) The youngest woman was of 22 years of age and the oldest was 45 years of the age. 


\section{Parity}

In the present study, 74 cases $(56.9 \%)$ of the cases were of 3rd Gravida. The highest gravidity in the study was 8 . 98 cases $(75.4 \%)$ of the cases were of 2 nd Parity and the highest parity was 7 .

\section{Registration status}

75 cases $(57.7 \%)$ were registered under our institute. 22 patients $(16.9 \%)$ were registered under BMC Hospitals and 21 patients $(16.2 \%)$ were registered under Private Practitioner. 7 patients $(5.4 \%)$ had come for Antenatal check-up only for a single time and 5 (3.8\%) were unregistered.

\section{Booked status}

78 patients $(60 \%)$ were booked under our institute. About 40 patients $(30.8 \%)$ were referred from periphery hospitals and 12 patients $(9.2 \%)$ had come on their own.

\section{Gestational age}

In the present study, 81 patients (62.3\%) had Term pregnancy i.e between 37 to $<42$ weeks of gestation. Post term pregnancies were $10(7.7 \%)$ whereas Preterm pregnancies were $39(30 \%)$. Out of these Preterm pregnancies, $22(16.9 \%)$ were late preterm and 17(13.1\%) were early preterm.

\section{Associated disorders}

In present study, 80 cases $(61.5 \%)$ were not associated with any disorder. Pregnancy Induced Hypertension(PIH) was the most common associated disorder seen in 24 patients $(18.5 \%)$. Diabetes mellitus was seen in 5 cases $(3.8 \%)$ whereas anaemia and Deranged Liver Function Test's (LFT'S) were seen in 3 cases each $(2.3 \%)$. Fever was seen in 3 patients $(2.3 \%)$ antenatally and two out of these three cases had Meconium intra-operatively. There was one case $(0.8 \%)$ of Tubal ligation (TL) Failure.

\section{Indication of LSCS}

The most common indication of LSCS in present study was foetal distress that is 53 cases $(40.8 \%)$ followed by Malpresentations 30 cases $(23.1 \%)$. PIH was seen in 24 cases $(18.5 \%)$.

Majority of the cases that is 35 cases $(66 \%)$ of foetal distress were associated with MSAF. Most common malpresentation noted in the study was Breech presentation that is 15 cases $(11.5 \%)$.

20 cases $(15.4 \%)$ showed Less Liquor either in the form of Oligohydramnios or anhydramnios. 18 cases $(13.8 \%)$ showed non-progress of labour (NPOL). Foeto-pelvic disproportion was seen in 8 cases $(6.2 \%)$. There were 4 cases $(3.1 \%)$ of bad obstetrics history $(\mathrm{BOH})$ (Figure 1$)$.

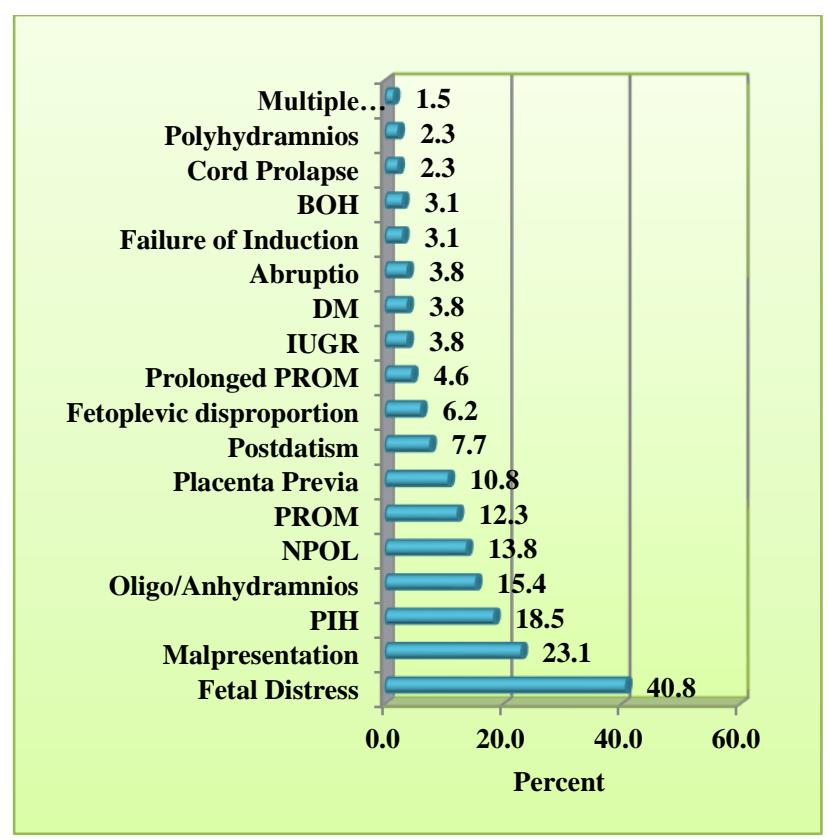

Figure 1: Indications of LSCS (N-130).

\section{Mode of identification of fetal distress}

As the major indication for caesarean section in multipara was fetal distress, it is studied in detail for its mode of identification. In the present study, majority of the cases are seen with Meconium Stained Amniotic Fluid (MSAF). There were 10 cases $(18.8 \%)$ with Fetal bradycardia and 4 cases $(7.5 \%)$ with Fetal Tachycardia. In 2 cases $(3.7 \%)$, Fetal distress was associated with Antepartum Haemorrhage (APH). In 2 cases (3.7\%), Non-Stress Test (NST) was non-reactive whereas variable deceleration was seen in 2 cases $(3.7 \%)$ and both these cases had tight loops of cord around neck. Out of the 29 cases that were taken for LSCS for Foetal distress only, with no MSAF pre-operatively, 11 cases showed MSAF intra-operatively.

\section{Intra-operative finding}

In the present study, most common intra-operative finding was Meconium Stained Amniotic Fluid (MSAF) i.e 35 cases. It is consistent with the most common indication of Caesarean section being Fetal distress. In 11 cases of Fetal distress, MSAF was found intra-operatively but not pre-operatively. The next most common intraoperative finding was Placenta previa i.e 14 cases. In case of malpresentations, breech was the most common intraoperative finding. Intra-operatively, Loop of Cord Around neck was found in 7 cases. The indications for LSCS in these 7 cases were Fetal distress ( 5 cases) and NPOL (2 cases).

\section{Intra-operative complication}

Post-Partum Hemorrrahge (PPH) -In the present study, 16 cases had PPH intra-operatively that is $12.3 \%$. Most of 
them were managed medically, although required blood/blood products transfusions. Uterine artery ligation was done in 4 cases and Obstetric Hysterectomy was done in 1 case. There was no injury to Bowel/Bladder in the caesarean sections neither were there any anaesthetic complications noted.

\section{Duration of surgery}

In the present study, the time taken from taking the patient on table to shifting her to recovery room after the procedure is noted. For majority of the cases, i.e 55 cases $(42.3 \%)$ it was between 60 to 90 minutes. Least amount of time taken was 28 minutes whereas maximum time was 160 minutes. It was taken in a caesarean section in which uterine artery ligation was done with exploration of broad ligament hematoma.

\section{Birth weight of neonates}

In this present study,33 of the neonates (25.4\%) were between the birth weight of 2.51 to $3.0 \mathrm{~kg}$. 57 neonates were Low Birth Weight. Out of them, 7 neonates (5.4\%) were Very Low Birth Weight and 3(2.3\%) were Extremely Low Birth Weight. 5 neonates were Macrosomic (3.8\%).

\section{Neonatal outcome}

In the present study, $60.8 \%$ neonates were with mother, whereas $38.5 \%$ neonates were admitted in NICU. There were $2.3 \%$ still births. There were $8.5 \%$ neonatal deaths (Figure 2).

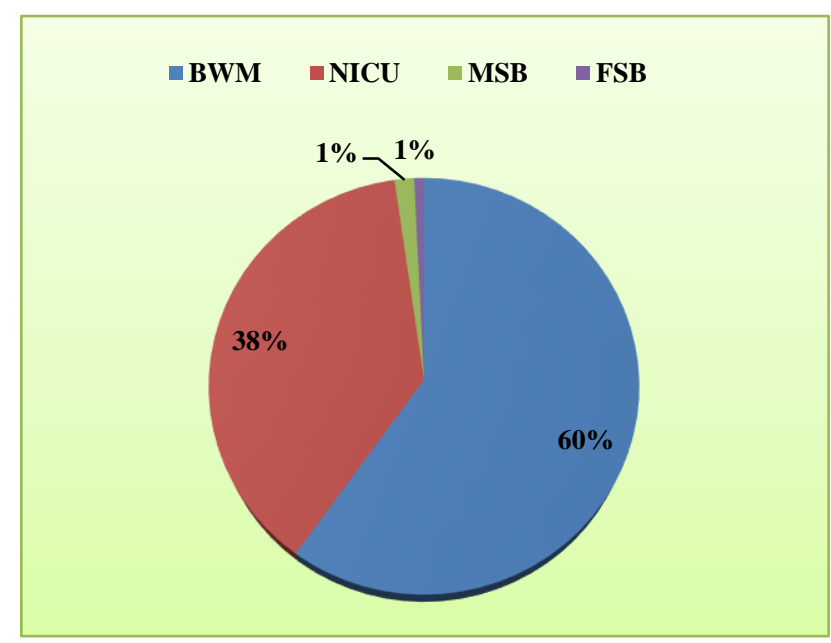

Figure 2: Neonatal outcome (N-132).

\section{Early perinatal morbidity and mortality}

In the present study 50 neonates were admitted in NICU. Major reason for the admission of the neonates in NICU was Respiratory Distress (RD) followed by Low Birth Weight (LBW). 9 cases were Large for Gestational age (LGA). 6 were admitted in view of Prolonged Rupture of
Membranes PPROM (PROM> 18 hours) and 5 for evaluation as they were born to Diabetic Mother (DM). Meconium aspiration was the reason for NICU admission in 4 neonates. Birth Asphyxia and Congenital anomalies accounted for 5 and 3 cases each. 2 neonates were initially given with mother but were later admitted in NICU in view of Jaundice. One neonate was admitted on day 6 and the second neonate was admitted on day 8 of life due to Jaundice. One neonate was admitted due to swelling in Prolapsed Hand (Figure 3). Birth asphyxia is seen in the neonates if they have an asphyxia insult proximate to the delivery that is severe enough to cause to acute neurological injury, (Apgar- 0 to 3 for 10 minutes, neurological manifestations such as seizure, coma, hypotonia, acidosis, multisystem organ dysfunction). ${ }^{5}$

\section{Early perinatal morbidity and mortality}

All the babies of birth asphyxia had neonatal death eventually.

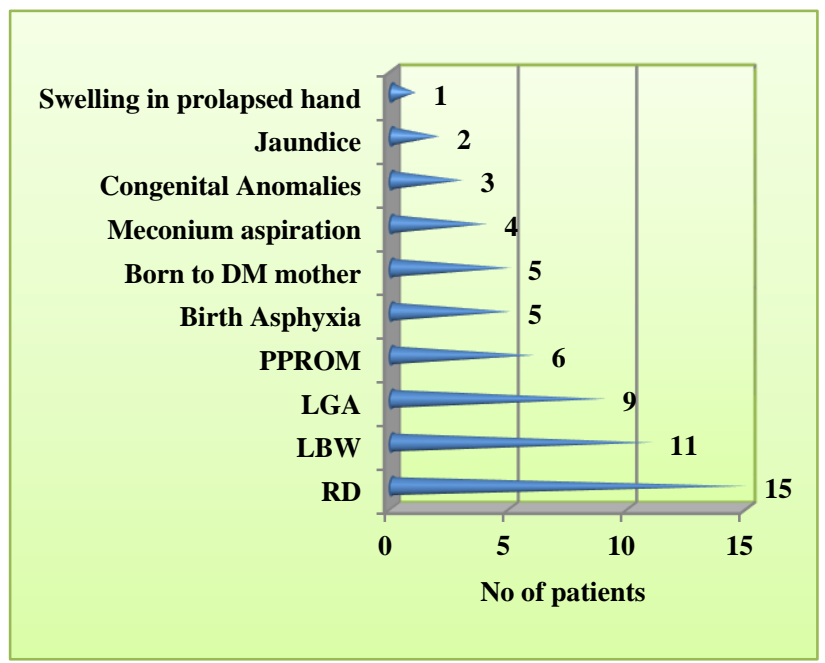

Figure 3: Early perinatal morbidity and mortality (N-50).

\section{Relation of indications and high-risk factors with neonatal morbidity}

In present study, highest morbidity is found in neonates of mothers who underwent LSCS for fetal distress with most common reason being respiratory distress in neonates. The $2^{\text {nd }}$ most common factor contributing to NICU admissions was PROM (Premature Rupture of Membranes). 6 neonates were admitted due to prolonged PROM ( $>18$ hours), in accordance with the policy for NICU admission in our institute and 5 due to respiratory distress (Figure 4). Out of 30 caesarean sections done in view of malpresentations, 14 neonates were admitted in NICU, mostly for birth asphyxia (3 cases) or congenital anomalies (3 cases). PIH was the next leading cause of NICU admission with majority of the neonates having respiratory distress (6 cases) and Low Birth weight (3 
cases). Out of 3 cases of cord prolapse one neonate was admitted in NICU for Respiratory distress. IUGR and feto-placental insuffiency resulted in 4 admissions in
NICU, out of which 3 were Low Birth Weight and 1 had respiratory distress.

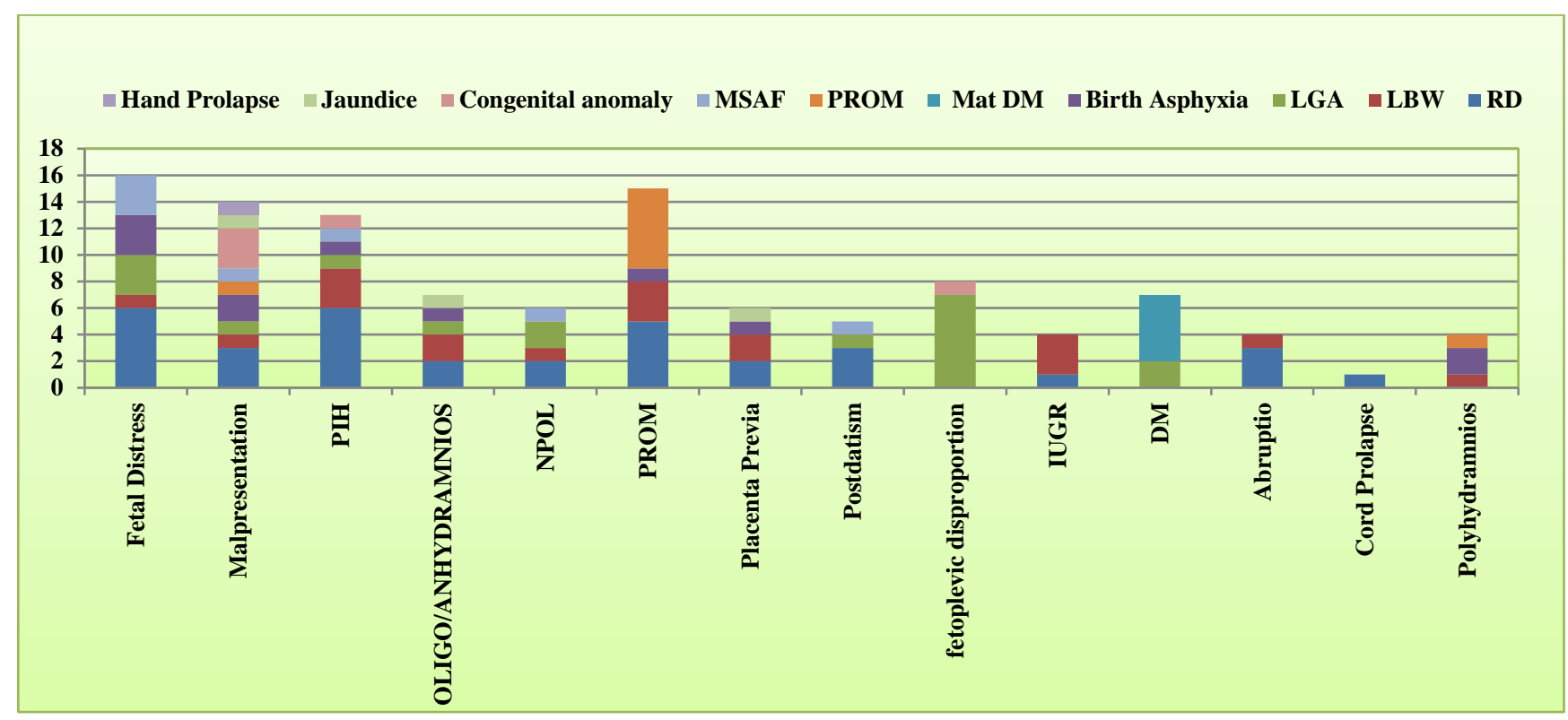

Figure 4: Relation of indications and high-risk factors with neonatal morbidity (n-50).

\section{Post-operative complications}

In the present study, major cause of Post-operative morbidity was wound gape, seen in $10.8 \%$ patients, that required Resuturing. 9 cases required Blood/Blood products transfusions in the post-operative period. 5 patients had Pyrexia. 2 patients had Wound discharge out of which 1 had pyrexia also. 1 case required Transfer to Critical Care unit for further management.

Out of 130 cases 15 patients required blood or blood products transfusions in either intra operative or postoperative period. The commonest associated risk factor in these patients was PIH with Abruptio Placentae.

\section{Stay in hospital}

In the present study, for $62(47.69 \%)$ of the patients the duration of hospital stay was between 7 to 14 days. There were 3 patients $(2.31 \%)$ who were admitted for more than 6 weeks. All 3 of them had prolonged Hospital Stay as the neonates were admitted in NICU for weight gain and preterm care.

\section{DISCUSSION}

There has been a steady increase in the rate of caesarean section in both developed and developing countries. Although the WHO recommends that there is no justification to increase a caesarean rate in excess of 10 to
$15 \%$, it may be difficult to contain the rates in tertiary institutes, catering to a large population of transferred cases. ${ }^{6}$ In the study done by Erika desai et al (2013) rate of primary Caesarean section in multipara was $29.5 \% .^{7}$ Saluja et al conducted a study in which the total caesarean section rate was $24.6 \%$ and rate of primary Caesarean section in multipara was $3.8 \%{ }^{8}$ Rao et al observed that total caesarean section rate and rate of primary Caesarean section in multipara were $29.4 \%$ and $10.2 \%$ respectively. ${ }^{9}$ In our present study, that total caesarean section rate was $30.7 \%$ and rate of primary Caesarean section in multipara was $23.7 \%$.

The most common indication of LSCS in present study was Foetal Distress i.e. 53 cases $(40.8 \%)$ followed by Malpresentations 30 cases $(23.1 \%)$.PIH was seen in 24 cases $(18.5 \%)$.

Majority of the cases i.e. 35 cases $(66 \%)$ of foetal distress were associated with MSAF. Most common malpresentation noted in the study was Breech presentation i.e. 15 cases (11.5\%). 20 cases (15.4\%) showed Less Liquor either in the form of Oligohydramnios or Anhydramnios. 18 cases(13.8\%) showed Non-progress of labour. Foeto-pelvic disproportion was seen in 8 cases $(6.2 \%)$. There were 4 cases $(3.1 \%)$ of $\mathrm{BOH}$. Pruthwiraj et al, Saluja et al and Rao et al in their study of primary caesarean section in multipara observed that most common indication was Malpresentations i.e. $23 \%, 30 \%, 33.5 \%$ respectively. 
Erika Desai Et al (2013) and present study have observed that the most common indication for Caesarean section was Fetal distress i.e. 25.5 and $40.7 \%$ respectively. ${ }^{10}$

In case of NICU admissions, there were total of 50 admissions out of 130 LSCS cases. Sethi Pruthwiraj et al (2010) in their study of primary caesarean section in multipara observed that $17 \%$ of the babies required NICU admissions. Also, Jyothi $\mathrm{H}$ Rao et al (2013) made a similar observation i.e. $16.5 \%$. Whereas in our present study $37.8 \%$ of the babies required NICU admissions. This may be because the leading indication of LSCS in present study is Fetal Distress as compared to Malpresentations being the leading cause in other studies.

Table 1: Total number of cases and most common indications of Primary LSCS in multipara in various studies.

\begin{tabular}{|c|c|c|c|}
\hline Author & $\mathbf{N}$ & Indication & $\%$ \\
\hline Pruthwiraj et al ${ }^{10}$ & 100 & Malpresentations & 23 \\
\hline Saluja et $\mathrm{al}^{8}$ & 50 & Malpresentations & 30 \\
\hline Desai et $\mathrm{al}^{7}$ & 86 & Fetal distress & 25.58 \\
\hline $\mathrm{Rao}_{\text {et }} \mathrm{al}^{9}$ & 200 & Malpresentations & 33.5 \\
\hline Present Study & 130 & Fetal distress & 40.7 \\
\hline
\end{tabular}

This high incidence of NICU admissions may also be because of high incidence of LBW and LGA requiring prophylactic NICU care in present study. Sethi Pruthwiraj et al in their study of primary caesarean section in multipara observed that Birth Asphyxia and pyrexia in babies were the most common cause of Perinatal morbidity i.e. NICU admissions (4\%), whereas in our present study Respiratory distress accounted for $30 \%$ of the NICU admissions.

\section{CONCLUSION}

From the above study it is very clear that many unforeseen complications occur in women who previously had a normal vaginal delivery. In our society, multiparity is a problem associated with poverty, illiteracy, ignorance and lack of knowledge of the available antenatal care and family planning methods. This result in a high birth rate and so many young women becomes grand multipara. This process causes short birth intervals, eventually jeopardizing the health of the mother and her offspring. This study reemphasizes the need of antenatal care and thorough care and vigilance in the management of labour. Though vaginal delivery is always safer than $\mathrm{C}$-section, difficult vaginal delivery and obstructed labour carries more morbidity and perinatal mortality when compared when compared to elective Csection. Previous vaginal delivery gives the patient as well as her relatives a false sense of security. In many cases, a caesarean becomes mandatory. The fact that a multipara has had one or more vaginal deliveries should be regarded as an optimistic historical fact, not as diagnostic criteria for spontaneous delivery of the pregnancy at hand.

Hence a multiparous woman in labour requires the same attention as that of primigravida. Primary caesarean in a multipara is a sensitive indicator of health delivery system. A multipara who has earlier delivered vaginally may still require a caesarean section for safe delivery. Good antenatal and intrapartum care and early referral will help reduce the maternal and perinatal morbidity.

Funding: No funding sources

Conflict of interest: None declared

Ethical approval: The study was approved by the Institutional Ethics Committee

\section{REFERENCES}

1. Cunningham. Leveno. Bloom. Houth. Rouse. Spong. Prenatal care.Williams Obstetrics $23^{\text {rd }}$ edition, 2009;8:194-5.

2. Basak S, Lahri D.Dystocia in eutocic multigravida. Journal Obstet Gynaecol India 1975;25:502-7.

3. Wilkinson C, McIlwaine G, Boulton-Jones C, Cole $\mathrm{S}$. Is a rising caesarean section inevitable. British $\mathbf{J}$ Obstet Gynaecol. 1996;105:45-52.

4. Kalavashishta, Rekha L, Gupta N.Primary caesarean section in grandmultipara. J Obstet Gynecol India 1972;26(4):651-57.

5. Cunningham. Leveno. Bloom. Houth. Rouse. Spong. Newborn infant. Williams Obstetrics 23rd edn, 2009,8:595

6. Asha Oumachigui : Changing Trends in Caesarean section. Obs and Gynaec Today. 2002; VII

7. Desai E, Leuva H, Leuva B, Kanani M.A study of primary caesarean section in multipara. Int J Reprod Contracept Obstet Gynecol. 2013; 2(3):320-4.

8. Saluja JK, Roy PK, Mahadik K. Study of primary caesarean section in multiparous women. Emerg. 2014;47:94.

9. Rao JH, Rampure N. "Study of Primary Caesarean Section in Multiparous Women".Journal of Evolution of Medical and Dental Sciences 2013;2(24):4414-8

10. Radhika T, Ficog Gs. Study of Primary Cesarean Section. In: Multigravidae. Global J Res Analy. 2018;6(11).

Cite this article as: Agrawal GP. Primary lower segment caesarean section in multipara: maternofetal outcomes. Int J Reprod Contracept Obstet Gynecol 2019;8:279-84. 